\title{
Research Paper: Comparing Motor Control Exercise and General Exercise on Lum- bo-Pelvic Stability of Chronic Nonspecific Low Back Pain Sufferers Using Endurance Stability Tests

\section{Mohammad Bagher Shamsi ${ }^{1}$, Morteza Saeb ${ }^{2},{ }^{*}$ Amir Hossein Hashemian ${ }^{3,4}$}

1. Department of Rehabilitation and Sport Medicine, School of Medicine, Kermanshah University of Medical Sciences, Kermanshah, Iran 2. Department of Orthopedics, School of Medicine, Kermanshah University of Medical Sciences, Kermanshah, Iran

3. Environmental Determinants of Health Research Center, Kermanshah University of Medical Sciences, Kermanshah, Iran.

4. Department of Epidemiology \& Biostatistics, Faculty of Health, Kermanshah University of Medical Sciences, Kermanshah, Iran.

\begin{tabular}{|c|c|}
\hline $\begin{array}{l}\text { Use your device to scan } \\
\text { and read the article online }\end{array}$ & CFtation: Shamsi MB, Saeb M, Hashemian AH. [Comparing Motor Control Exercise and General Exercise on Lumbo-Pelvic \\
\hline 口iftip & Stability of Chronic Nonspecific Low Back Pain Sufferers Using Endurance Stability Tests (Persian)]. Archives of Rehabilitation. \\
\hline Prysir & 2018; 18(4):306-315. https://doi.org/10.21859/jrehab.18.4.5 \\
\hline 口ithy & dol": https://doi.org/10.21859/jrehab.18.4.5 \\
\hline
\end{tabular}

Received: 12 Jul. 2017 Accepted: 04 Nov. 2017
Keywords: Low back pain, Motor control exercise, General exercise, Endurance test

\section{A B STRACT}

Objective There is a controversy regarding whether Motor Control Exercise (MCE) is more effective than General Exercise (GE) for Chronic Low Back Pain (CLBP). MCE has been compared to other exercises using outcomes such as pain, but they have not been compared with outcomes related to lumbopelvic stability. As it is claimed that MCE enhances back stability, endurance (as a component of stability) tests that assess lumbopelvic stability were selected. The aim of this study was to compare MCE and GE on CLBP patients based on lumbopelvic stability through three endurance tests.

Materials \& Methods This study was conducted in Rasool Akram hospital and Motor Control Lab of School of Rehabilitation Science. While being admitted, 43 people (aged 18-60 yrs) with chronic nonspecific Low Back Pain (LBP) were alternately allocated to either motor control $(n=22)$ or general exercise group ( $n=21$ ). Inclusion criteria included having LBP for more than three months and pain intensity 3-6 in the Visual Analogue Scale. Exclusion criteria included having pathology or anomaly lower limb such as hyperplasia, inflammatory diseases, sever osteoporosis, arthritis or other bone diseases. Labeling patients as CLBP was based on physical examination and clinical tests. Three endurance score stability tests such as: Trunk flexor, Trunk extensor, and side bridge tests used as primary outcomes. Pain and disability were also measured as secondary variables at two levels, prior to the start and end of the program. The MCE and GE programs of 16 sessions each were provided to the two groups of participants. Both groups performed their exercises three times a week. In the MCE group, anatomy and function of local back stabilizing muscles and the way they could be activated were taught. For the GE group, exercises activating the extensor (paraspinals) and flexor (abdominals) muscle groups were used.

Results At the beginning of the study, there was no significant difference in the variables of test time, pain intensity, and disability index. After the intervention, test times increased, and disability and pain decreased within the groups. There was no significant difference between two groups in increasing test times $(\mathrm{P}=0.23$ to 0.36 ) or decreasing disability and pain, $\mathrm{P}=0.16, \mathrm{P}=0.73$ respectively.

Conclusion Though it is claimed that MCE increases spinal stability, the lack of significant difference in the results of variables between the two groups may be due to our MCE not being specific for increasing spinal stability or equal effects of both interventions on increasing spinal stability and no preference of MCE. It may also be due to poor sensitivity of our outcome measures in detecting changes in spinal stability, especially with respect to the sample size. Based on these results, it could be concluded that MCE is not more effective than GE in improving endurance core stability tests and reducing disability and pain in chronic non-specific LBP patients.

\section{* Corresponding Author:}

Amir Hossein Hashemian, PhD

Address: Department of Epidemiology \& Biostatistics, Faculty of Health, Kermanshah University of Medical Sciences, Kermanshah, Iran Tel: +98 (918) 1337135

E-Mail: dr.ahashemian@kums.ac.ir 


\title{
مقايسه تمرينهاى كتترل حركتى مهرهها و تمرينهاى عمومى در بايدارى استقامتى ناحيه

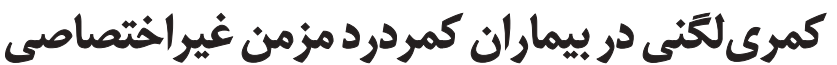

\author{
محمدباقر شمسى'، مرتضى صائب'، "اميرحسين هاشميان" \\ 1- كرووه توانبخشى وطب ورزشي، دانشكده يزشكى، دانشكاه علوم يزشكى كرمانشاه، كرمانشاه، ايران.

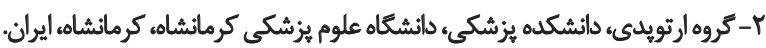

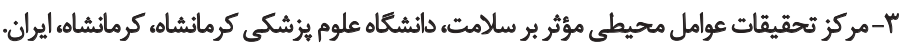

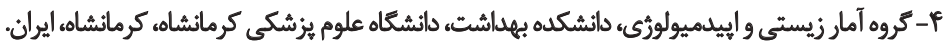

\section{حكיie}

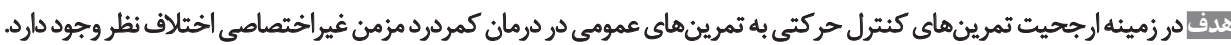

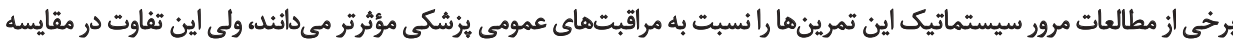

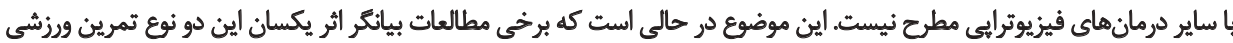

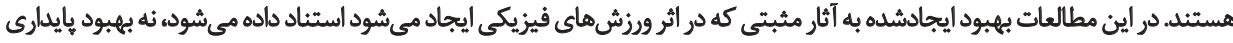

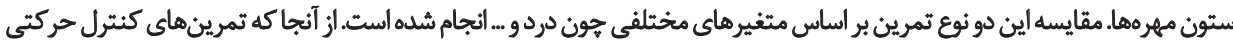

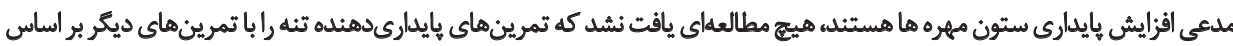

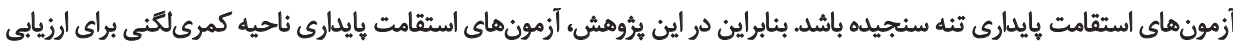

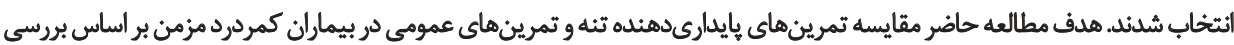

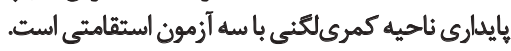

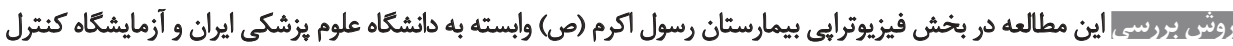

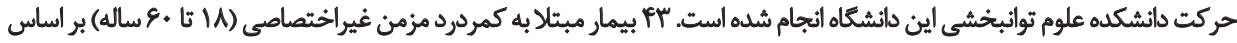

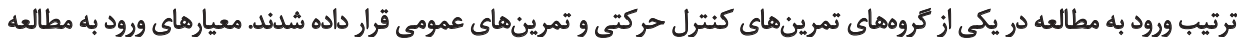

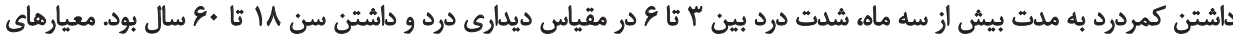

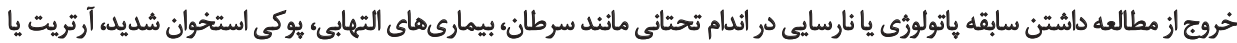

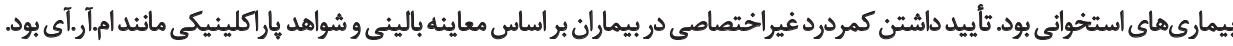

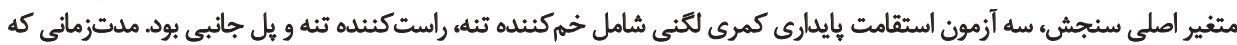

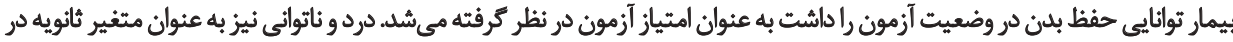

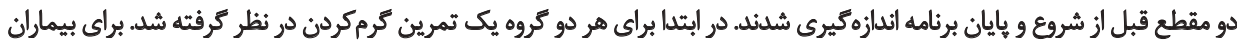

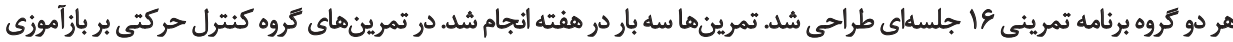

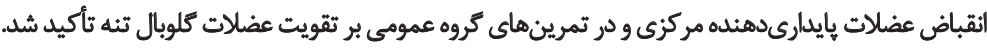

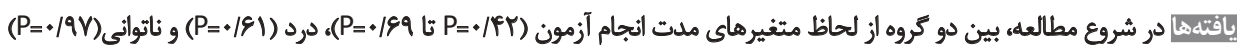

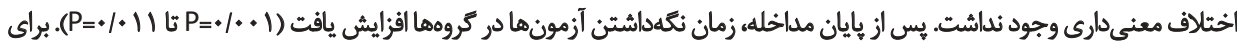

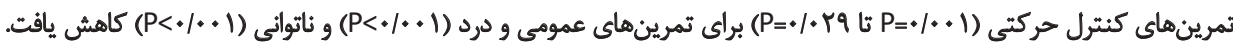

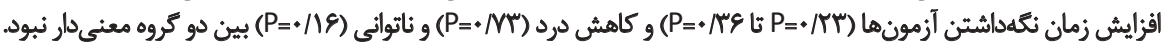

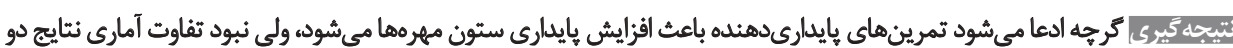

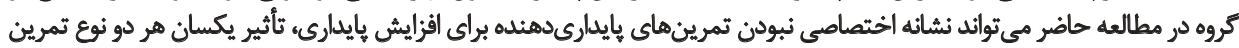

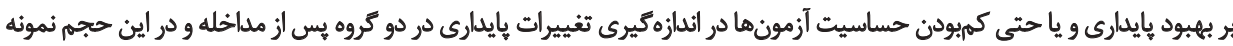

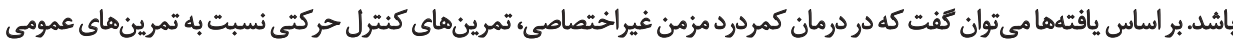

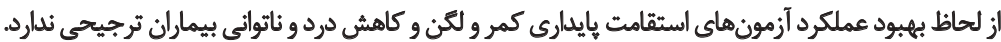

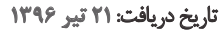

تاريخ بذيرش: تآ تبان
كليدوازوها:

كمردرد، تمرين كاي

كنترل حركتي، تمردين

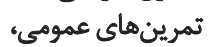
آزمونهاي استقامث عمومي 


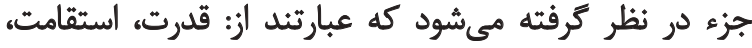

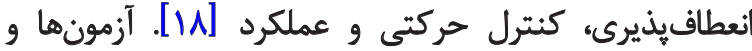

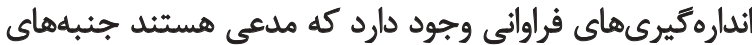

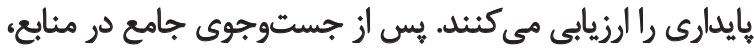

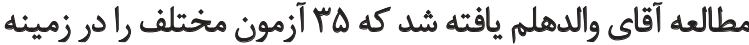

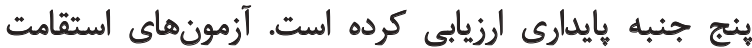

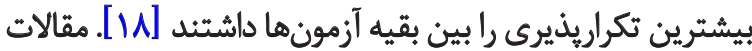

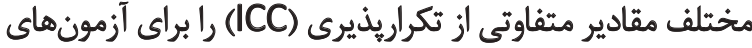

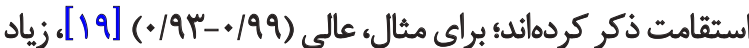

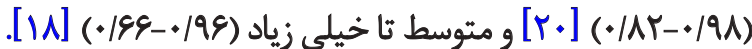

آزمونهاى استقامت آزمونهايى هستند كه شخص ئيك

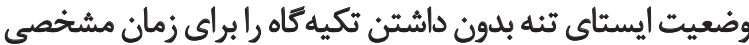

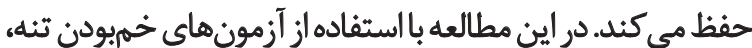

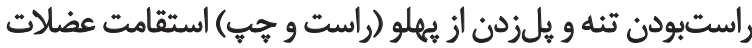

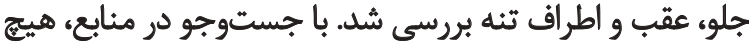

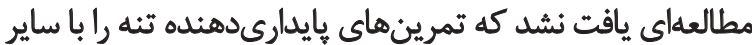

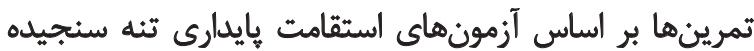

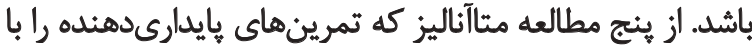

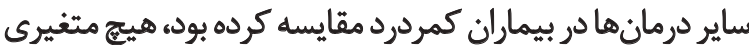

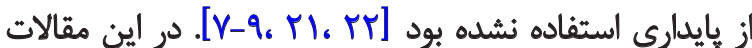

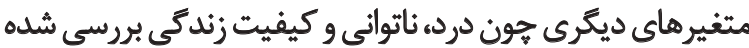

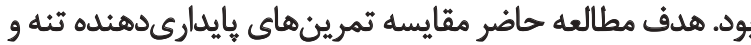

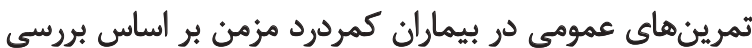

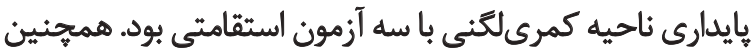

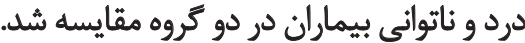

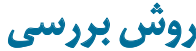

اين هطالعه از نوع كارآزمايي بالينى شبهتجربيى بود. تأييديه

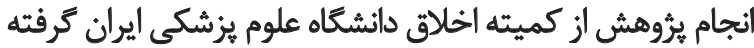

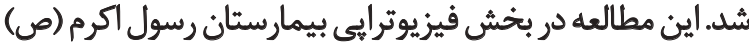

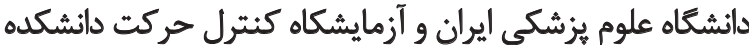
علوم توائبخشى اين دانشعاه انجام شده است.

بر مبناي مطالعات مشابه، براي تشخيص اختلاف ها ثانيهاي

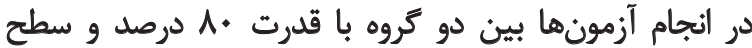

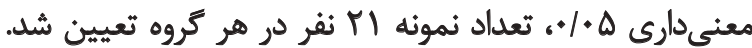

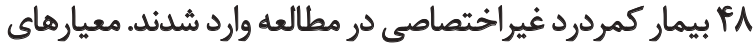

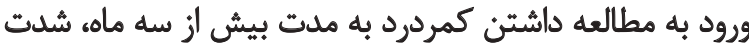

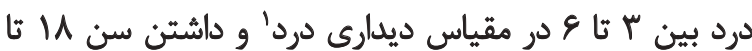

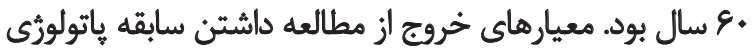

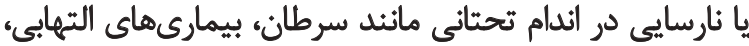

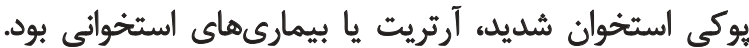

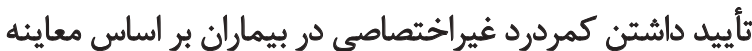

1. Visual Analogue Scale (VAS)
مقدمه

درصد زيادى از بيماران كمردرد كه علت خاصى براي درد آنها

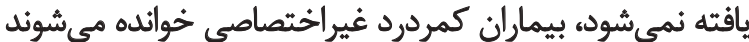

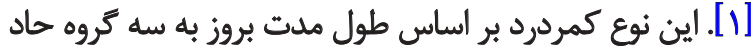

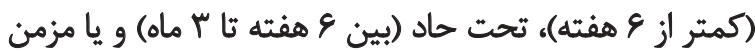

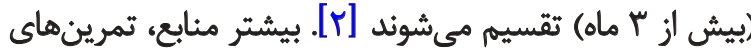

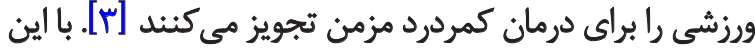

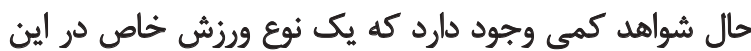

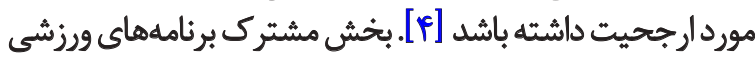

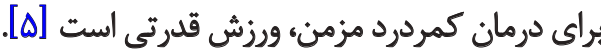
تمرينهاى ورزشى يايدارى دهنده مركزى درمانهاى جديدى

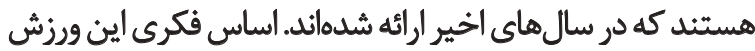

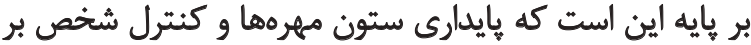

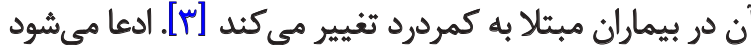

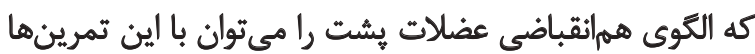

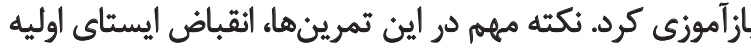

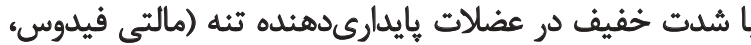

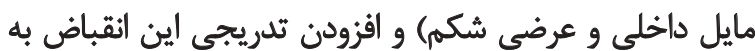

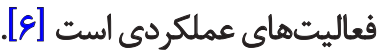

ارجحيت تمرينهاى يايدارىدهنده نسبت به تمرينهاى

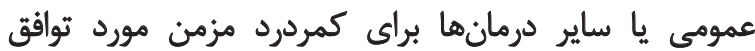

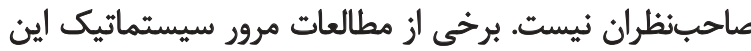

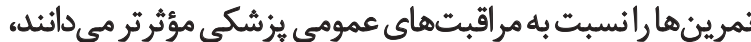

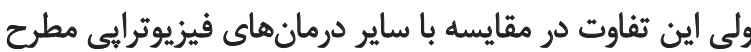

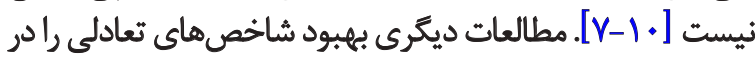

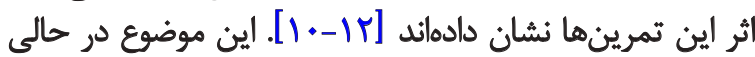

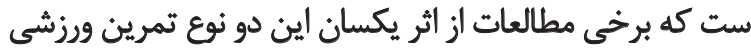

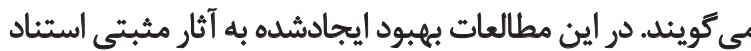

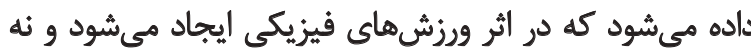

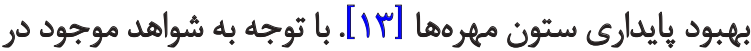

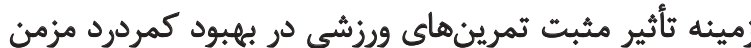

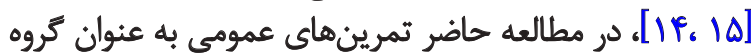

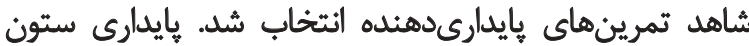

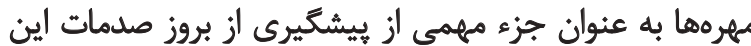

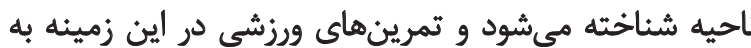

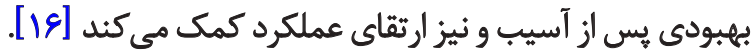
كيايدارى ناحيه كمرى لكنى عبارت است ازئ توانايى شخص در

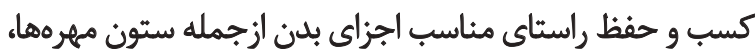

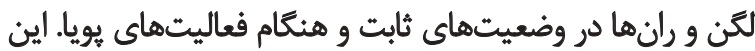
بايدارى توسط بافتهاى غيرانقباضي (مائند رباطها) و وانقان انقباض

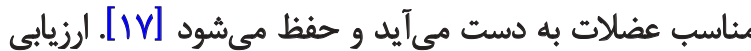

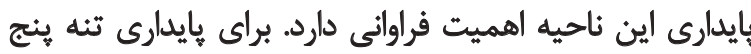


جلسه متوالى يا ها جلسه متثاوب غيبت داشتند از مطالعه خارج

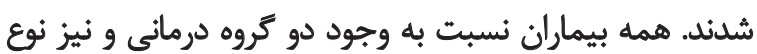

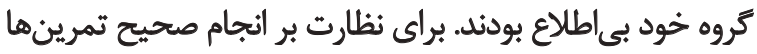

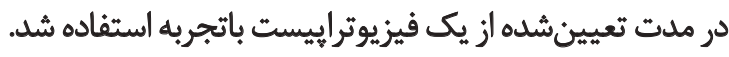

\section{أزمون هاى استقامت}

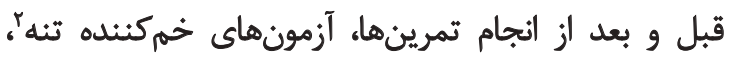

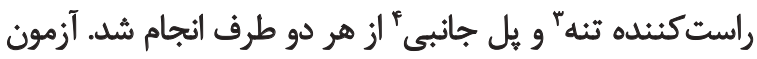

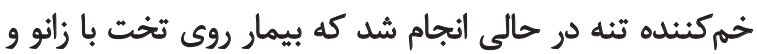

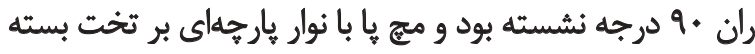

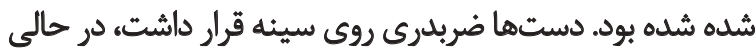

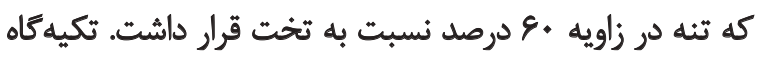

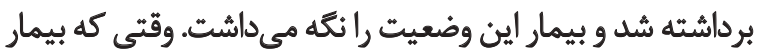

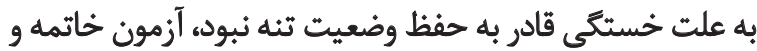

$$
\text { زمان آن ثبت مىشد (تصوير شماره (). }
$$

آزمون راست كنئده تنه در حالى انجام شد كله بيمار در وضعيت

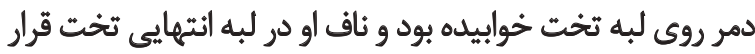

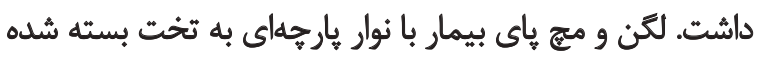

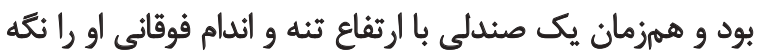

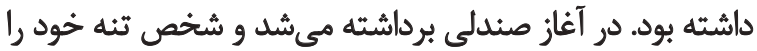

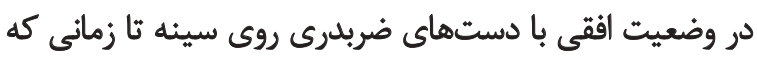

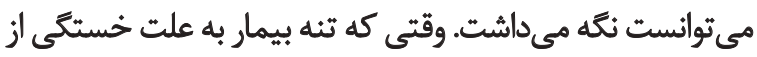

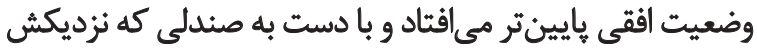
قرار داشت تكيه مي داد آزمون خاتمه مىيافت (تصوير شماره Y). آزمون ئل جانبي در حالت خوابيده به بيهلو با زانوهاى راست

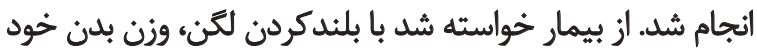

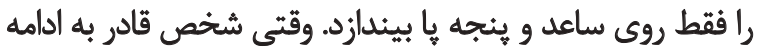

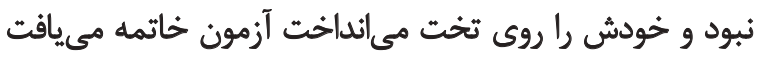

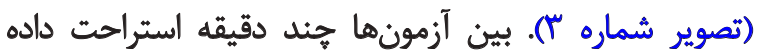

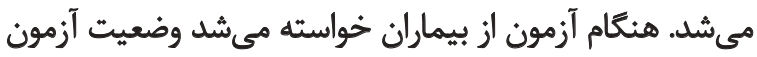

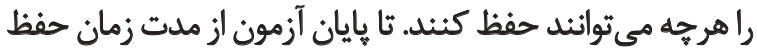

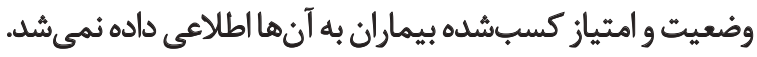

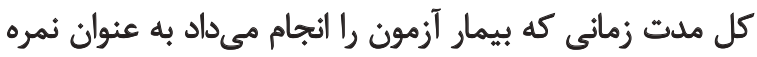

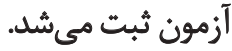

قبل و بعد از تمرينها، شدت درد بيماران با مقياس ديدارى دئ بدري

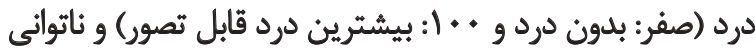

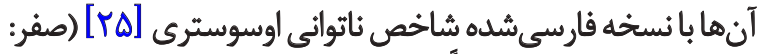

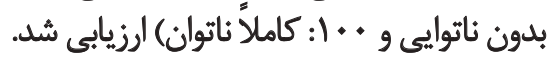

براى مقايسه مدت انجام آزمونها، ناتوانى و درد بين دو كروه

2. Trunk Flexor Test

3. Trunk Extensor Test

4. Side Bridge Test

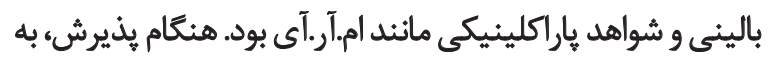

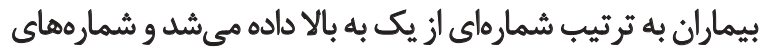

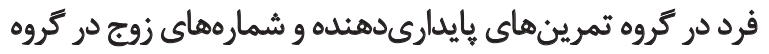

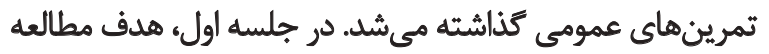

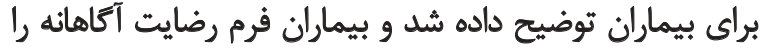
امضا كردند. مراحل انجام كار در تصوير شماره f آمده است

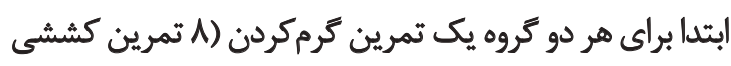

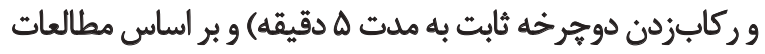

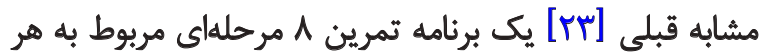

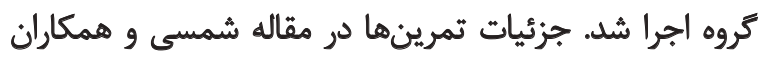

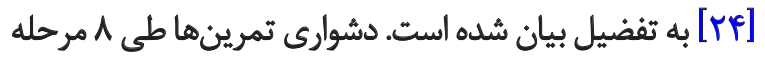

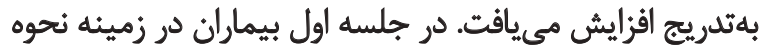

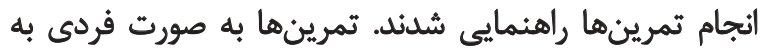

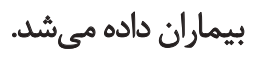

\section{كروه تمرين هاى بايداري دهنده مركزى}

در اين گروه توضيحاتى درباره تمرينها به بيماران داده شد.

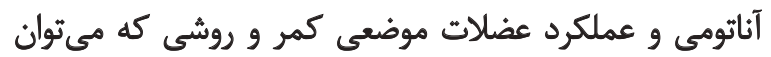

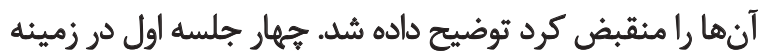

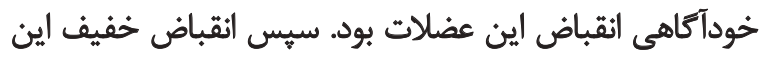

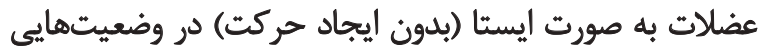

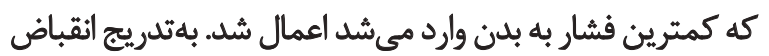

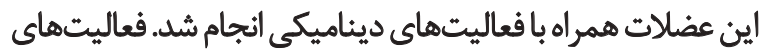

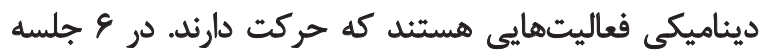

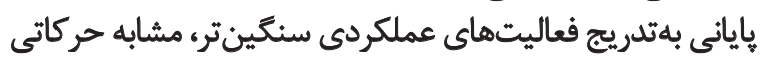

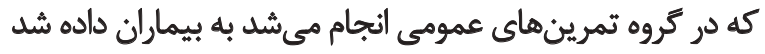

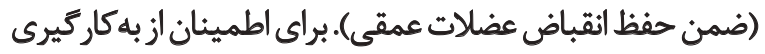

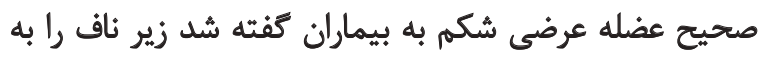

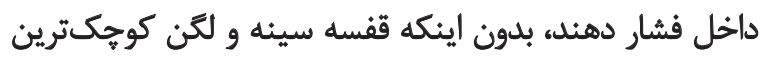

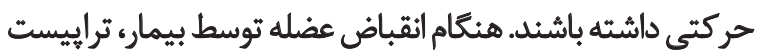

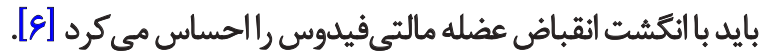

$$
\text { يروه تمرينهاي عمومي }
$$

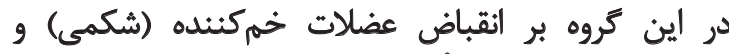

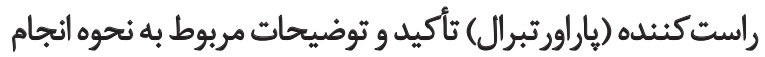

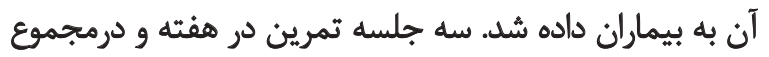

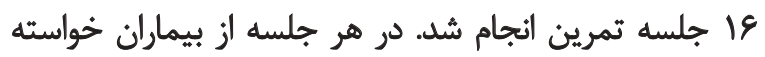

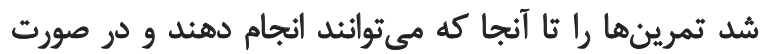

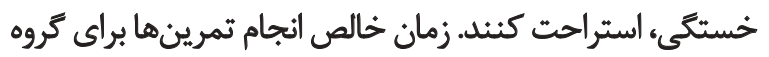

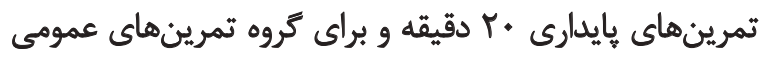

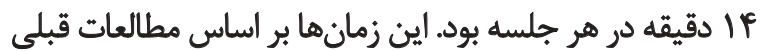

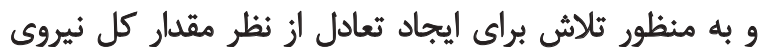

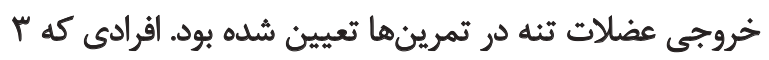


وجو

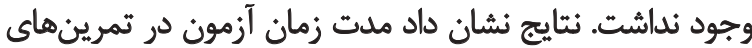

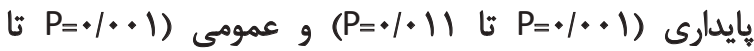

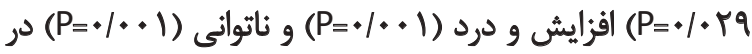

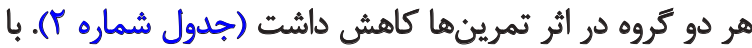

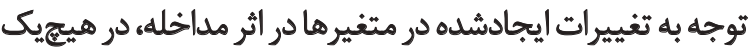

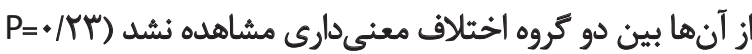

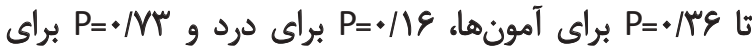

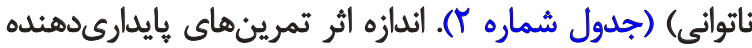

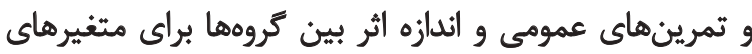
مختلف در جدول شماره Y آمده است.

بحث

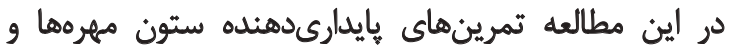

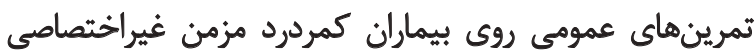

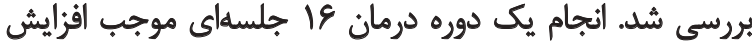

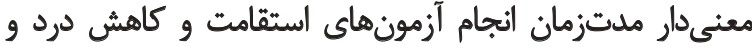
ناتوانى در هر دو گروه شل، ولى هيج تفاونى آمونى بين دو كروه نشان ندان نداد.

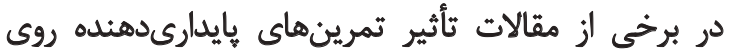

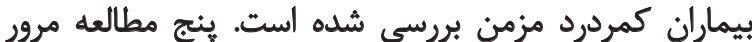

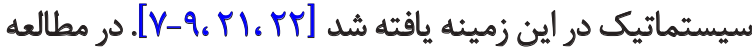

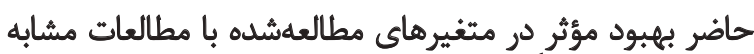

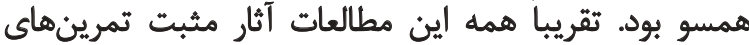

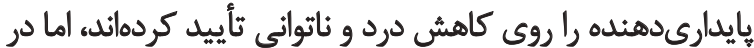

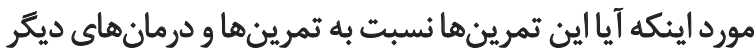

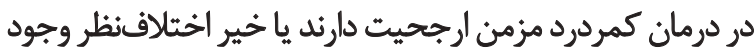

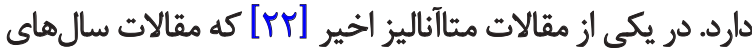

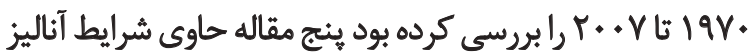

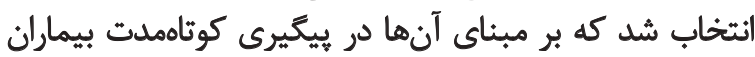

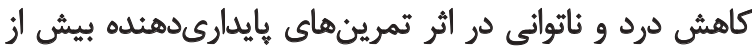

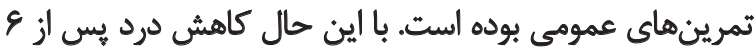

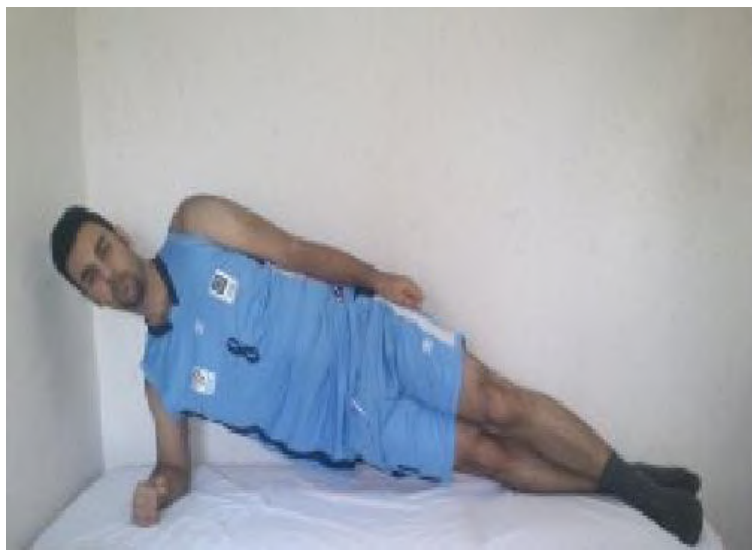

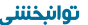

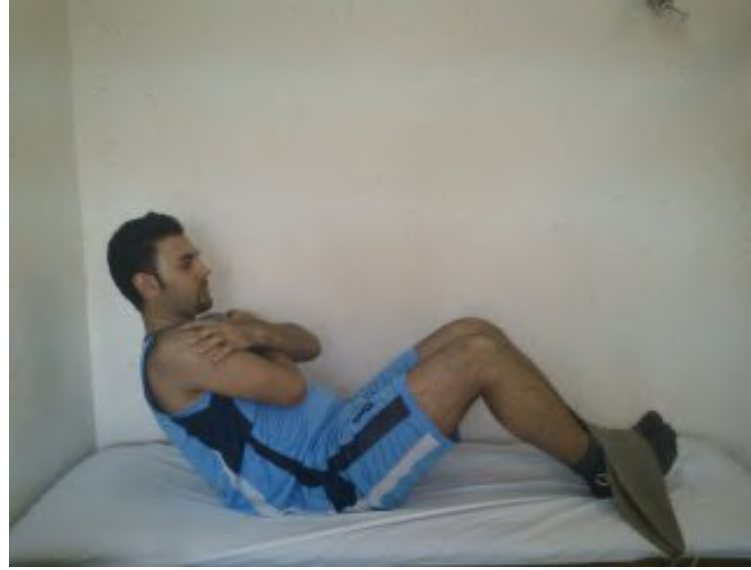

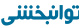

تصوير الـ آزمون خهمكينده تئ

هنكام شروع مطالعه براى اطمينان از يكسانبودن نمونهها و در

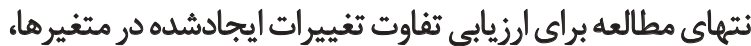

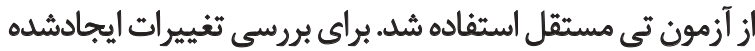

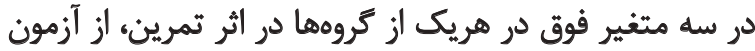

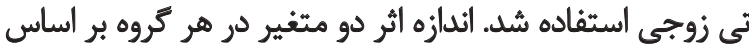

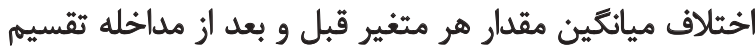

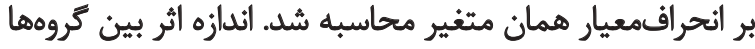

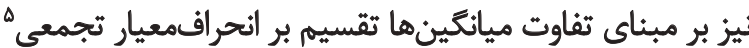

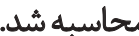

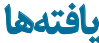

ينج نفر از بيماران به علت غيبت بيش از حلد مجاز از مطالعه

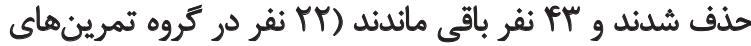

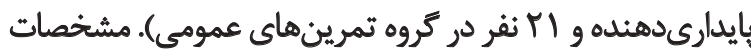

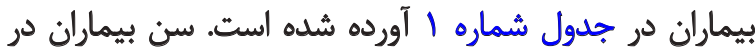

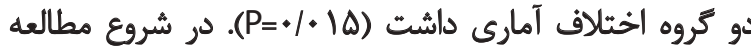

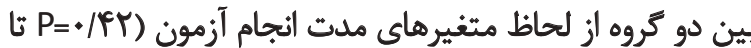

\section{Pooled Standard Deviation}

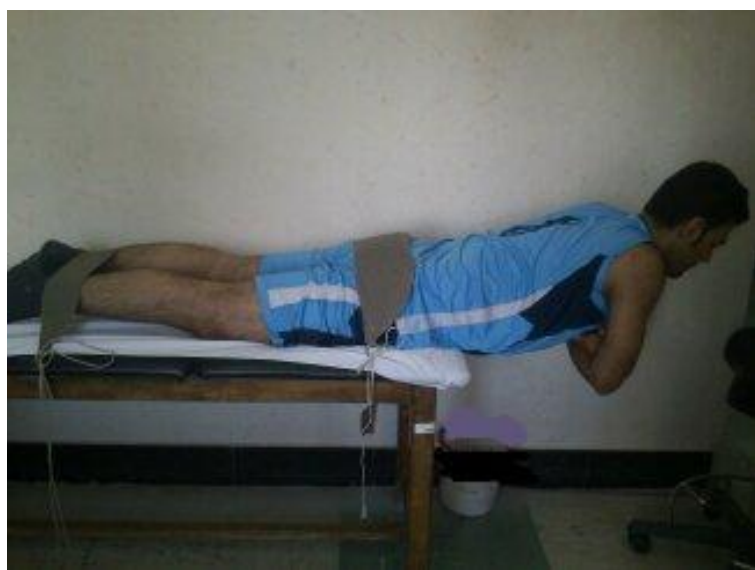

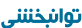

تصوير ب. آزمون راستكثنده تثنه 


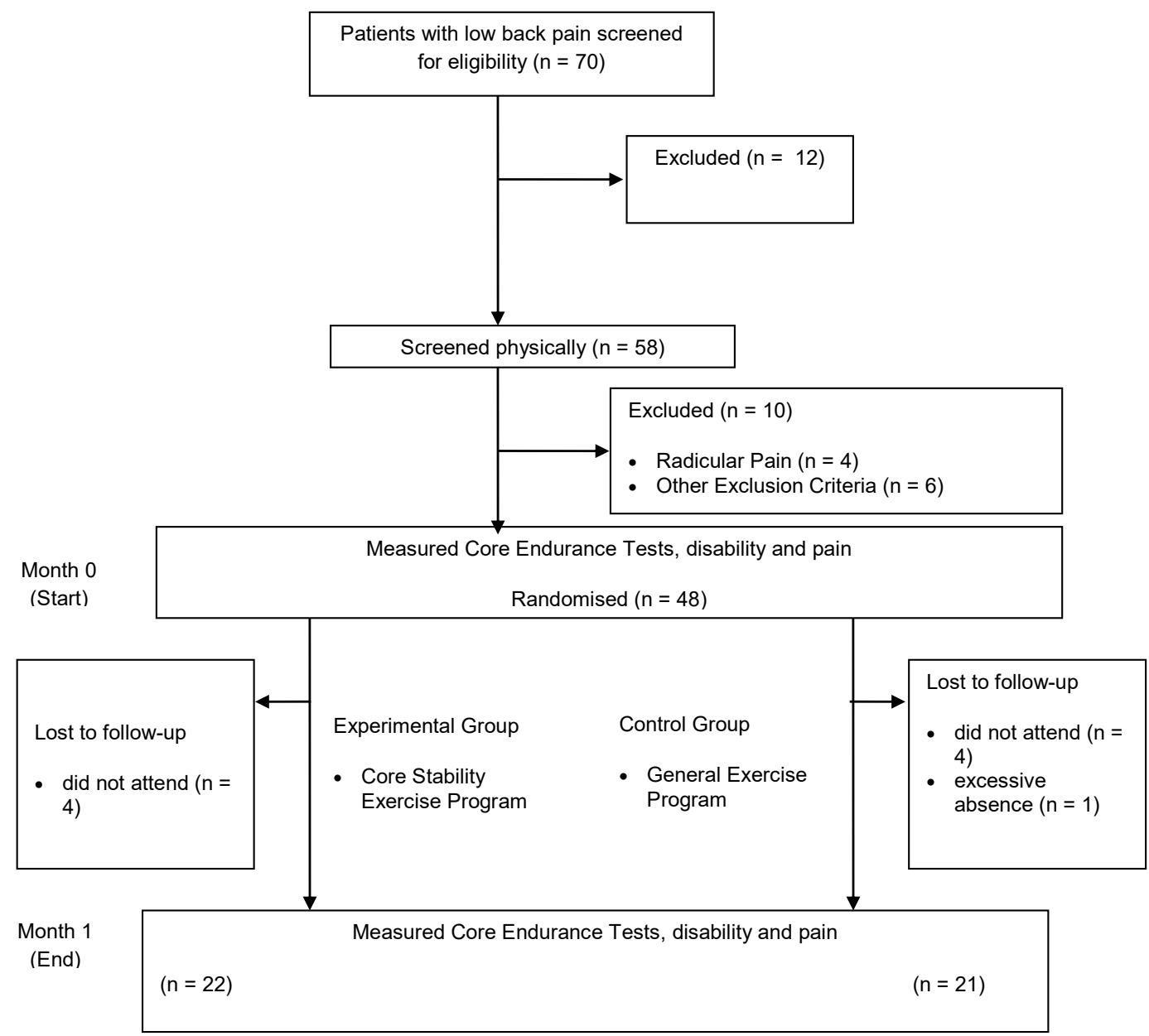

توانبخننى

تصوير ع. مراحل شركت نمونهها در يُروهش

و كنترل (سيستم عصبى). صدمه يا عدم استفاده از هر يك از

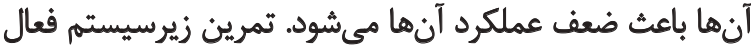

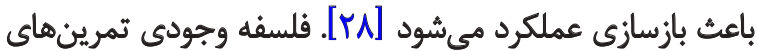

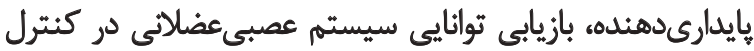

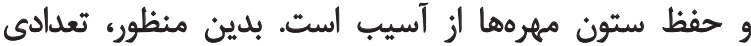

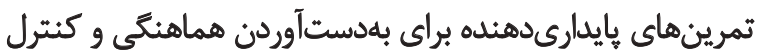

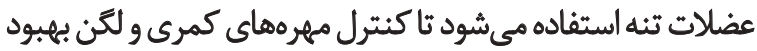

تا Y Iاه بين دو تروه تفاوت معنى دارى نداشت. در اين مطالعه

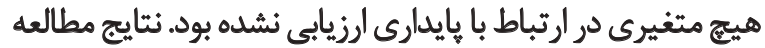

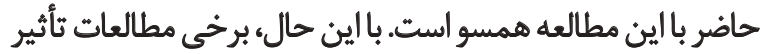

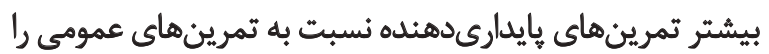

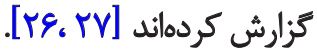

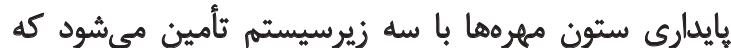

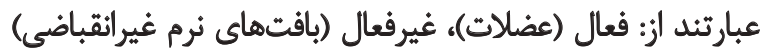

جدول ا. اطلاعات جمعيتشناختى بيماران

\begin{tabular}{|c|c|c|}
\hline تروه تمرينهاى كتترل حركتى & تروه تمرينهاى عمومى & مشخصات \\
\hline$\checkmark$ & 8 & 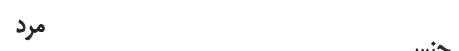 \\
\hline 10 & 10 & ij \\
\hline $\operatorname{mar}(11 / M)$ & $\operatorname{PV} /(1 . / \pi)$ & سن(سال) / ميانكين (انحرافمعيا)) \\
\hline $\operatorname{ses} /(Q /)$ & $\mid E T / V(N))$ & قدارسانتىمثر)/ ميانكين (انحر|فمعيار) \\
\hline$V \cdot / 1(18 / 1)$ & $n \in / r(1 . / 0)$ & وزن (كيلوكرم)/ ميانكين (انحرافمعيار) \\
\hline
\end{tabular}

توانبخننى 
جدول r. ميانكين (انحرافمعيار)، اندازه اثر، اختلاف داخل كروه و بينَّروهى متغيرهاي مطالعهشهه در دو گروه متعاقب يك دوره درمان

\begin{tabular}{|c|c|c|c|c|c|c|c|c|c|c|}
\hline \multirow[b]{2}{*}{ بين ئروهيى اثئ } & \multirow{2}{*}{ اختّالاف بين مقدل } & \multicolumn{4}{|c|}{ كروه تمرينهاي عموهى } & \multicolumn{4}{|c|}{ كروه تمرين هاى كتترل حركتي } & \multirow[b]{2}{*}{ متغير ييامد } \\
\hline & & أدازه & اختمال & بعد & قبل & اثدازه & اختمال & بعد & قبل & \\
\hline .89 & $P=118$ & $1 / M$ & $P=* 1 *+1$ & $\operatorname{VV} / 8(1 . / 9)$ & $\Delta \cdot N(11 / M)$ & $V / \Delta V$ & $P<+1.01$ & $\pi / A(1 \cdot / \Delta)$ & $\Delta \cdot / \Delta(1 Y / M)$ & شاخوس نائوانى \\
\hline - MAY & $P \equiv \cdot M^{\mu}$ & $r / M$ & $P<1 . .+1$ & $\mid \Delta / /(I r / A)$ & $\Delta T / Q(q / 0)$ & r/T" & $P<.1 .01$ & $18 / 1(11 / 1)$ & $\Delta 1 / F($ V(A) & درد \\
\hline$+/ \%$ & $P=\star \pi T$ & $+/ 4 Y$ & $P=.1 .79$ & ENH $(E \& / \cdot)$ & $m / F(r \varepsilon / q)$ & $\cdot \mathbb{A}$ & $\mathrm{P}=. / . .1$ & $91 / \Delta(8 \& / 8)$ & PNITVIA) & أزمون خثيم كنئده تثنه \\
\hline$r / .4$ & $P=\cdot \pi$ &.$/ 9 x$ & $P=* / . .1$ & $\| \Psi / T(\Delta T / 0)$ & $A \cdot / A(\mp N F)$ & $\cdot N 9$ & $P=* / . .1$ & $\| V / r(8 \cdot / T)$ & $V \cdot / E(\Delta V / 1)$ & آزمون راستكنئه \\
\hline$+1 \Delta 8$ & $\mathrm{P}=+/ \pi_{*}$ & +194 & $P<+\infty 1$ & $F y / *(T V / q)$ & $r \Delta / \Gamma(T T / \pi)$ & $+/ 4+$ & $\mathrm{P}=* /+11$ & $\operatorname{TT/T}(T N T)$ & rVIr(T\&/N) & آزمون يل عانرضيه بها \\
\hline + & $\mathrm{P}=+/ \mathrm{F}$ & $+19 \mathrm{~N}$ & $\mathrm{P}=+1 \cdot+1$ & $P V / *(T Y / q)$ & $r E / r(r+/ \Lambda)$ & +181 & $\mathrm{P}=* / \omega 1$. & $R T / T(T N T)$ & $M / T(T N I)$ & 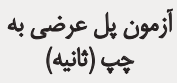 \\
\hline
\end{tabular}

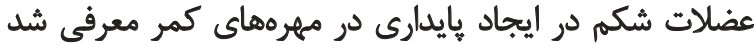

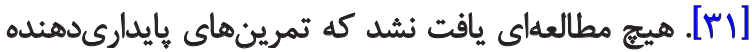

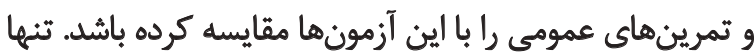

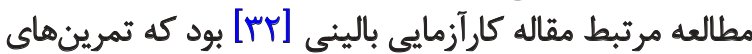

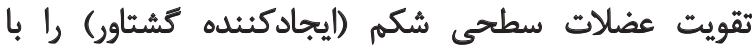

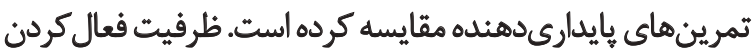

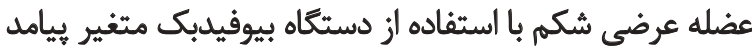

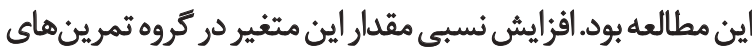
بايداري دهنده مشههود بود.

هيجيك از مطالعات در زمينه تمرينهاى بإيدارى دهنده، رابطه

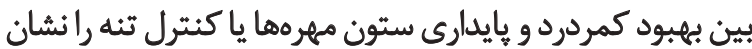

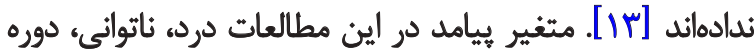

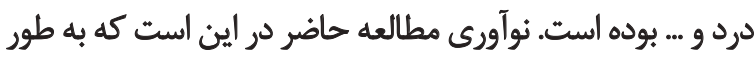

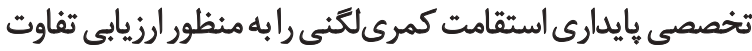
در تأثير تمرينهاي انجامشده براى كمردرد ارزيابي مي كنئد

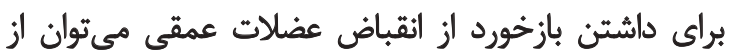

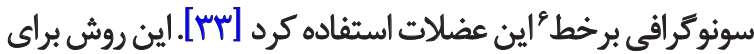

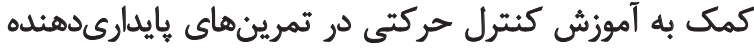

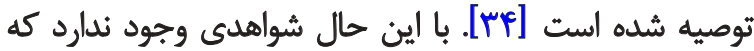

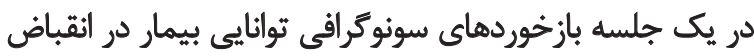

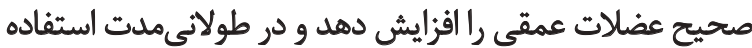

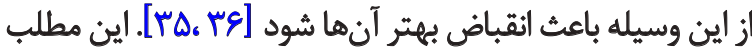

\section{Real-time}

يابد. همجنين بازيابي ظرفيت (قدرت و استقامت) عضلات تنه براى

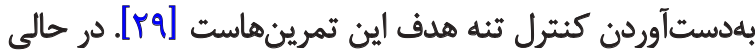
كه تمركز اين تمرينها بر باز آموزى عضلات عمقى تند تنه است، با اين حال برخى از صاحبنظران معتقدند عضلات تنه (و نه

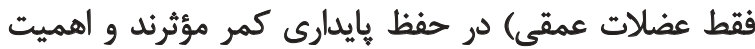

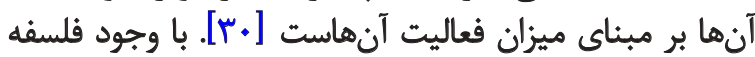

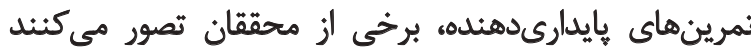

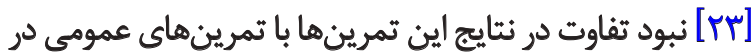

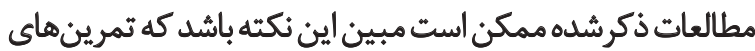

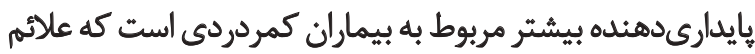

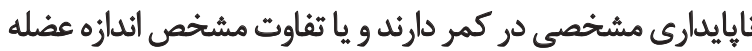

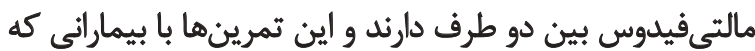
هيج علامتى از نايايداري بالينى ندارند ارتباطي ندارين ندارد. كرجه ادعا مىشود تمرينهاى קايدارىدهنده باعث افزايش

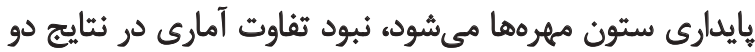

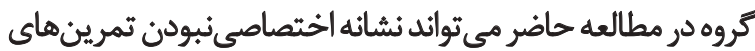

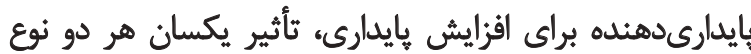

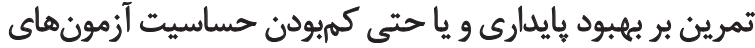

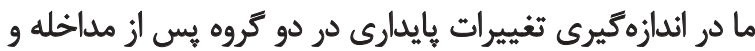

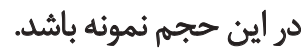

آزمونهاى زيادى جنبههاى مختلف بإيدارى تثنه را ارزيابى

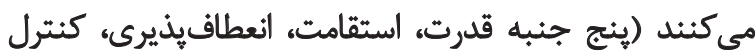

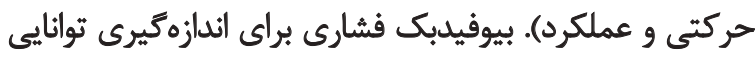


كه انجام تمرينهاى يايدارى با سونوكرافى منجر به نتايج بهتر مي شود بررسى نشده است. تثيجه در درمان كمردرد مزمن غيراختصاصى، هر دو نوع تمرينهاى



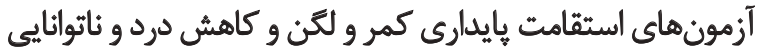
بيماران مؤثر بودند، ولى هيج يك بر ديكرى ترئ ترجيح ندارد.

$$
\text { تشكر وقدردانى }
$$

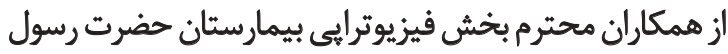

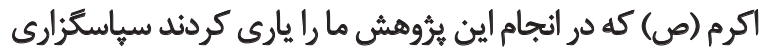

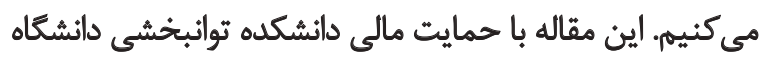

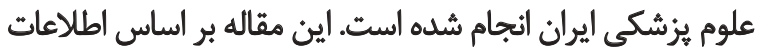

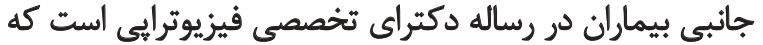

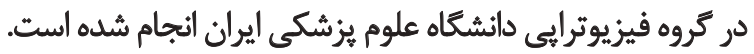




\section{References}

[1] Van Tulder M, Koes B. Evidence-based medicine for low back pain. Medical Radiology. 2007; 111-25. doi: 10.1007/978-3-54068483-1_5

[2] Refshauge KM. Low back pain investigations and prognosis: A review. British Journal of Sports Medicine . 2006; 40(6):494-8. doi: 10.1136/bjsm.2004.016659

[3] Costa LOP, Maher CG, Latimer J, Hodges PW, Herbert RD, Refshauge KM, et al. Motor control exercise for chronic low back pain: a randomized placebo-controlled trial. Physical therapy. 2009; 89(12):1275-86. doi: 10.2522/ptj.20090218

[4] Van Tulder M, Malmivaara A, Esmail R, Koes B. Exercise therapy for low back pain. Spine. 2000; 25(21):2784-96. doi: 10.1097/00007632-200011010-00011

[5] Liddle SD, Baxter DG, Gracey JH. Exercise and chronic low back pain: What works. Pain. 2004; 107(1):176-90. doi: 10.1016/j. pain.2003.10.017

[6] Richardson CA, Hodges P, Hides JA. Therapeutic exercise for lumbopelvic stabilization. New York: Churchill Livingstone; 2004.

[7] Rackwitz B, de Bie R, Limm H, von Garnier K, Ewert T, Stucki G. Segmental stabilizing exercises and low back pain, What is the evidence: A systematic review of randomized controlled trials. Clinical Rehabilitation. 2006; 20(7):553-67. doi: 10.1191/0269215506cr977oa

[8] Ferreira PH, Ferreira ML, Maher CG, Herbert RD, Refshauge K. Specific stabilisation exercise for spinal and pelvic pain: A systematic review. Australian Journal of Physiotherapy. 2006; 52(2):79_ 88. doi: 10.1016/s0004-9514(06)70043-5

[9] Macedo LG, Maher CG, Latimer J, McAuley JH. Motor control exercise for persistent, nonspecific low back pain: A systematic review. Physical Therapy. 2008; 89(1):9-25. doi: 10.2522/ ptj.20080103

[10] Okhovatian F, Kahrizi S, Samadi-Pour A. [Comarison between three common remedial exercises in pain severity of patients with mechanical CLBP (Persian)]. Archives of Rehabilitation. 2003; $4(2): 7-15$

[11] Salehi S, Hedayati R, Bakhtiari A H, sanjari M A, Ghorbani R. [The comparative study of the effect of stabilization exercise and stretching-strengthening exercise on balance parameters in forward head posture patients (Persian)]. Archives of Rehabilitation. 2013; 14(1):50-60

[12] Ya'ghoubi Z, Kahrizi S, Parnian-Pour M, Ebrahimi-Takmajani E, Faghih-Zadeh S. [The short effects of two spinal stabilization exercise on balance tests and limit of stability in men with nonspecific chronic low back pain: Randomized clinical trial study (Persian)]. Archives of Rehabilitation. 2012; 13(1):102-113.

[13] Lederman E. The myth of core stability. Journal of Bodywork and Movement Therapies. 2010; 14(1):84-98. doi: 10.1016/j. jbmt.2009.08.001

[14] Hayden JA, van Tulder MW, Tomlinson G. Systematic review: Strategies for using exercise therapy to improve outcomes in chronic low back pain. Annals of Internal Medicine. 2005; 142(9):776. doi: 10.7326/0003-4819-142-9-200505030-00014

[15] Henchoz Y, Kai-Lik So A. Exercise and nonspecific low back pain: A literature review. Joint Bone Spine. 2008; 75(5):533-9. doi: 10.1016/j.jbspin.2008.03.003

[16] Willardson JM. Core stability training. Journal of Strength and Conditioning Research. 2007; 21(3):979-85. doi: 10.1519/00124278-200708000-00054

[17] Perrott MA, Pizzari T, Opar M, Cook J. Development of clinical rating criteria for tests of lumbopelvic stability. Rehabilitation Research and Practice. Hindawi Limited; 2012; 2012:1-7. doi: $10.1155 / 2012 / 803637$

[18] Waldhelm A, Li L. Endurance tests are the most reliable core stability related measurements. Journal of Sport and Health Science. 2012; 1(2):121-8. doi: 10.1016/j.jshs.2012.07.007

[19] McGill SM, Childs A, Liebenson C. Endurance times for low back stabilization exercises: Clinical targets for testing and training from a normal database. Archives of Physical Medicine and Rehabilitation. 1999; 80(8):941-4. doi: 10.1016/s0003-9993(99)90087-

[20] Evans K, Refshauge KM, Adams R. Trunk muscle endurance tests: Reliability, and gender differences in athletes. Journal of Science and Medicine in Sport. 2007; 10(6):447-55. doi: 10.1016/j. jsams.2006.09.003

[21] Hauggaard A, Persson AL. Specific spinal stabilisation exercises in patients with low back pain: A systematic review. Physical Therapy Reviews. 2007; 12(3):233-48. doi: 10.1179/108331907x222949

[22] Wang X-Q, Zheng J-J, Yu Z-W, Bi X, Lou S-J, Liu J, et al. A meta-analysis of core stability exercise versus general exercise for chronic low back pain. PLoS ONE. 2012; 7(12):52082. doi: 10.1371/journal.pone.0052082

[23] Koumantakis GA, Watson PJ, Oldham JA. Trunk muscle stabilization training plus general exercise versus general exercise only: Randomized controlled trial of patients with recurrent low back pain. Physical therapy. 2005; 85(3):209-25. doi: 10.1093/ ptj/85.3.209

[24] Shamsi MB, Sarrafzadeh J, Jamshidi A. Comparing core stability and traditional trunk exercise on chronic low back pain patients using three functional lumbopelvic stability tests. Physiotherapy Theory and Practice. 2014; 31(2):89-98. doi: 10.3109/09593985.2014.959144

[25] Mousavi SJ, Parnianpour M, Mehdian H, Montazeri A, Mobini B. The oswestry disability index, the roland-morris disability questionnaire, and the quebec back pain disability scale: Translation and validation studies of the Iranian versions. Spine. 2006; 31(14):454-9. doi: 10.1097/01.brs.0000222141.61424.f7

[26] O'Sullivan PB, Phyty GDM, Twomey LT, Allison GT. Evaluation of specific stabilizing exercise in the treatment of chronic low back pain with radiologic diagnosis of spondylolysis or spondylolisthesis. Spine. 1997; 22(24):2959-67. doi: 10.1097/00007632-199712150-00020

[27] Ferreira ML, Ferreira PH, Latimer J, Herbert RD, Hodges PW Jennings MD, et al. Comparison of general exercise, motor control exercise and spinal manipulative therapy for chronic low back 
pain: A randomized trial. Pain. 2007; 131(1):31-7. doi: 10.1016/j. pain.2006.12.008

[28] Mills JD, Taunton JE, Mills WA. The effect of a 10-week training regimen on lumbo-pelvic stability and athletic performance in female athletes: A randomized-controlled trial. Physical Therapy in Sport. 2005; 6(2):60-6. doi: 10.1016/j.ptsp.2005.02.006

[29] Hodges PW. Core stability exercise in chronic low back pain. Orthopedic Clinics of North America. 2003; 34(2):245-54. doi: 10.1016/s0030-5898(03)00003-8

[30] McGill SM. Low back stability: From formal description to issues for performance and rehabilitation. Exercise and Sport Sciences Reviews. 2001; 29(1):26-31. doi: 10.1097/00003677200101000-00006

[31] Jull G, Richardson C, Toppenberg R, Comerford M, Bui B. Towards a measurement of active muscle control for lumbar stabilisation. Australian Journal of Physiotherapy. 1993; 39(3):187-93. doi: 10.1016/s0004-9514(14)60481-5

[32] França FR, Burke TN, Hanada ES, Marques AP. Segmental stabilization and muscular strengthening in chronic low back pain: A comparative study. Clinics. 2010; 65(10):1013-7. doi: 10.1590/ s1807-59322010001000015

[33] Hodges PW, Pengel LHM, Herbert RD, Gandevia SC. Measurement of muscle contraction with ultrasound imaging. Muscle \& Nerve. 2003; 27(6):682-92. doi: 10.1002/mus.10375

[34] Richardson C. Therapeutic exercise for spinal segmental stabilization in low back pain: Scientific basis and clinical approach. New York: Churchill Livingstone; 1999.

[35] Henry SM, Westervelt KC. The use of real-time ultrasound feedback in teaching abdominal hollowing exercises to healthy subjects. Journal of Orthopaedic \& Sports Physical Therapy. 2005; 35(6):338-45. doi: 10.2519/jospt.2005.35.6.338

[36] Teyhen DS, Miltenberger CE, Deiters HM, Del Toro YM, Pulliam JN, Childs JD, et al. The use of ultrasound imaging of the abdominal drawing-in maneuver in subjects with low back pain. Journal of Orthopaedic \& Sports Physical Therapy. Journal of Orthopaedic \& Sports Physical Therapy. 2005; 35(6):346-55. doi: 10.2519/jospt.2005.35.6.346 